\title{
Une approche située et critique du concept de gamification
}

\section{Maude Bonenfant et Sébastien Genvo}

\section{OpenEdition}

\section{Journals}

Édition électronique

URL : http://journals.openedition.org/sdj/286

DOI : $10.4000 /$ sdj.286

ISSN : 2269-2657

Éditeur

Laboratoire EXPERICE - Centre de Recherche Interuniversitaire Expérience Ressources Culturelles Education

\section{Référence électronique}

Maude Bonenfant et Sébastien Genvo, «Une approche située et critique du concept de gamification », Sciences du jeu [En ligne], 2 | 2014, mis en ligne le 24 octobre 2014, consulté le 27 mars 2021. URL : http://journals.openedition.org/sdj/286 ; DOl : https://doi.org/10.4000/sdj.286

Ce document a été généré automatiquement le 27 mars 2021.

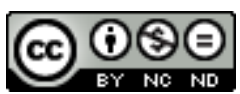

La revue Sciences du jeu est mise à disposition selon les termes de la Licence Creative Commons Attribution - Pas d'Utilisation Commerciale - Pas de Modification 4.0 International. 


\title{
Une approche située et critique du concept de gamification
}

\author{
Maude Bonenfant et Sébastien Genvo
}

1 Depuis plusieurs années, la propagation et la multiplication des formes de jeu numérique au sein de différents secteurs d'activité sont accompagnées par l'émergence de plusieurs concepts et cadres théoriques pour décrire, analyser, voire développer ce phénomène. Dans la recherche académique anglophone, dans le milieu du markéting et dans les pratiques professionnelles de conception de jeux, le concept de gamification s'est rapidement diffusé. Parfois traduit en français par le terme de « ludification », il est défini par Zichermann et Cunningham (2011) comme un processus qui consiste à user de l'état d'esprit et de la mécanique du jeu pour résoudre des problèmes et faire participer les usagers, les principes de base du design de jeu étant appliqués dans différents contextes. Dans le cadre de cette introduction, nous verrons que ce concept est à la fois pertinent pour penser le monde contemporain, car il participe de plusieurs enjeux, mais il soulève aussi de nombreuses questions, entre autres en ce qui concerne ses différents sens, acceptions et traductions. Cela incite à le mettre à distance pour mieux en apprécier la portée et, éventuellement, envisager des réponses aux questions soulevées ainsi que des alternatives. Dans un premier temps, nous verrons ses contextes d'usage puis, dans un second temps, nous tenterons de définir le concept afin d'évaluer, dans un dernier temps, certains effets de son application, particulièrement dans le domaine économique.

\section{Les contextes d'usage}

2 Un des premiers contextes dans lequel l'expression gamification a fait son apparition est celui du markéting alors qu'elle y est présentée comme une "solution miracle » pour les compagnies. Il faut ainsi comprendre qu'au cœur de la gamification se trouve la question de la motivation et de "l'engagement » d'un individu dans diverses activités qui reposent sur l'emploi « des structures de récompense, des renforcements positifs et boucles de feedback subtiles en même temps que des mécanismes comme des 
points, des médailles, des niveaux, des challenges et des tableaux de leaders " (Zichermann \& Cunningham, 2011, ix, traduction libre). Il faut cependant mentionner que Gabe Zichermann et Chistopher Cunningham donnent un sens particulier à la notion d'engagement dans leur définition de la gamification, ce qui permet parallèlement d'en saisir certaines finalités : " le terme "engagement", dans un sens "business", indique la connexion entre un consommateur et un produit ou un service [...]. Plutôt que l'idée antique de pousser le consommateur à "acheter plus", engager l'utilisateur afin de générer des revenus est le modèle markéting du futur. Dit plus simplement, l'engagement ne suit pas les revenus. À la place, après l'engagement, les revenus suivent " (Zichermann, Cunningham, 2011, xiii, traduction libre). Dans ce contexte, l'engagement ne concerne pas l'individu ou le citoyen, mais bien le consommateur qui $\left(s^{\prime}\right)$ investit auprès d'une entreprise par le biais de stratégies ludiques.

3 Initialement, il s'agit essentiellement de développer à travers le concept de gamification de nouvelles techniques de commercialisation pour renforcer la fidélisation des consommateurs/utilisateurs/joueurs. Par exemple, plusieurs compagnies utilisent des stratégies ludiques de markéting auxquelles les usagers du cyberespace se sont habitués pour présenter plus ou moins directement leurs produits (Slush Puppie, Century 21, Master Card et bien d'autres). Elles invitent les participants à collaborer, à s'entraider, à s'échanger des items, à comparer leurs résultats, afin d'atteindre les objectifs inscrits dans la conception de la plateforme, du site, du jeu vidéo, de l'installation, etc. Réciproquement, les plateformes de socialisation en ligne sont envahies de jeux dans lesquels on fait la promotion de divers produits : par exemple, le jeu The Sims Social, affilié au réseau social Facebook, invite les joueurs à essayer des produits " virtuels » de marques connues (Samsung, Toyota, Dunkin Donuts, Dove,...).

4 Or, si la gamification est particulièrement présente dans le markéting, elle apparaît dans d'autres domaines. Selon Zichermann et Cunningham, tout contexte serait potentiellement adapté à la gamification, puisque ce sont les « mécanismes » du jeu - et non son thème - qui vont procurer $\mathrm{du}$ « fun ». On en trouve quelques exemples parmi des jeux en ligne à succès récents, comme le jeu sur réseau social Farmville (Zynga, 2009), où plusieurs millions de joueurs s'affairent à des tâches banales telles que planter leur potager et aménager leur ferme, ou encore Flight control (Firemint, 2009) qui propose de gérer le trafic aérien. Les auteurs font à ce titre la comparaison avec les machines dans les casinos : ce ne serait pas tant le décor de la machine qui importerait que sa machinerie sous-jacente (pousser le bouton, tirer sur le bras, attendre que les trois cerises soient alignées pour gagner). Toute situation, même la plus simple, quotidienne et a priori ennuyante, serait donc susceptible d'être traduite selon les principes de la gamification. Raison pour laquelle la notion recouvre parfois de manière très large l'acception d'une ludification de l'éducation, le domaine pédagogique faisant fréquemment appel à des stratégies ludiques qui peuvent mener jusqu'aux jeux sérieux.

En ce sens, Jane McGonigal (2011) prétend que les jeux vidéo peuvent aussi être utiles pour améliorer le sort du monde et défendre des causes sociales en usant de la gamification dans des contextes nouveaux comme celui des déplacements et de la consommation de pétrole (World Without Oil, 2007). Selon elle, les joueurs excellent à résoudre des problèmes en collaborant, avantage qu'il est possible de mettre à profit pour trouver des solutions à des problèmes sociaux concrets en usant du jeu pour 
présenter ces problématiques. La coopération entre des joueurs a d'ailleurs été exploitée dans le domaine des sciences, alors que la mise en forme ludique de problèmes scientifiques a rendu possible leur résolution. Le journal Nature révèle, par exemple, qu'un jeu vidéo en ligne multijoueur a permis de déchiffrer les structures d'une protéine (Cooper et al., 2010). La possibilité de collaborer encourage non seulement le pouvoir des joueurs dans la résolution de problèmes, mais aussi l'attrait que les structures ludiques peuvent avoir sur les internautes lorsqu'il est question de compétition. L'intérêt pour les interactions entre les participants, le " plaisir partagé " et la socialisation en ligne pourraient ainsi expliquer en grande partie l'usage croissant des stratégies ludiques.

\section{Définir la notion}

On remarque que, si la gamification est principalement associée au monde économique, cette notion est en fait beaucoup plus large et recouvre de nombreux contextes et significations. En ce sens, elle est régulièrement questionnée et critiquée, notamment pour sa conception béhavioriste du game design. À ce titre, Sebastian Deterding (2010) relève qu'un jeu n'est pas intéressant simplement parce qu'il est un jeu, mais avant tout parce qu'il est bien conçu. L'adjonction d'un système de points ou de récompenses ne peut suffire à faire d'une situation un jeu, qui plus est un jeu intéressant. Deterding réfute à ce titre que le thème du jeu n'aurait aucune incidence dans son attrait (Deterding, 2011). Le jeu en ligne Progress Wars ${ }^{1}$ (Jakob Stjerning, 2010) peut être vu comme une caricature de la gamification pour démontrer par l'absurde l'ennui généré par une application stricte de ses principes : au sein d'une page web austère, il suffit à l'internaute de cliquer sur un bouton pour faire progresser une barre d'accumulation de points. Au bout de quelques clics, le joueur passe un niveau et obtient un niveau supplémentaire. Il recommence alors à cliquer sur le bouton pour gagner un nouveau niveau, etc. Dans le domaine du markéting, on pourrait, de la même manière, présenter le cas des cartes de fidélité et des points bonis (dont les points Air Miles constituent un excellent exemple) pour démontrer que cette stratégie de gamification n'est en rien un jeu satisfaisant, mais plutôt un mode de surveillance, de contrôle social et un biopouvoir (Coll, 2014).

7 La position de certains auteurs est alors de redéfinir les soubassements théoriques de la notion sans pour autant changer un terme qui connaît à présent une certaine fortune d'usage. C'est notamment le propos d'un texte de Deterding, Dixon, Khaled et Nacke (2011) dont nous proposons une version française pour ouvrir le dossier de ce numéro. Par ailleurs, dans l'introduction de l'ouvrage collectif Rethinking gamification (Fuchs, Fizek, Ruffino, Schrape, 2014), une approche plus globale du terme est proposée à côté de l'acception "markéting ", où il s'agit d'insister sur un processus général où les jeux et les expériences ludiques sont compris comme une composante essentielle de nos sociétés. Dans cette perspective il s'agit de « regarder comment les pratiques et rituels, appartenant à différents contextes historiques et culturels, peuvent prendre forme ou ressembler à des jeux $»^{2}$ (Ibid. : 7). Il y aurait aussi lieu d'indiquer que d'autres auteurs, comme Ian Bogost (2011), appellent à forger des cadres théoriques alternatifs permettant de penser et nommer différemment les processus de mutation et de contagion du jeu. Il y a en ce sens un intérêt à repositionner la notion par rapport à d'autres concepts qui abordent des phénomènes similaires, que ce soit pour souligner 
leurs équivalences ou leurs dissemblances. À ce titre, il faut indiquer que l'étude de l'avènement du jeu dans des domaines dont on pensait qu'ils lui étaient étrangers n'est pas récente.

On trouve en effet plusieurs pistes à ce sujet dans l'ouvrage Homo ludens. Essai sur la fonction sociale du jeu de Johan Huizinga (1938), ouvrage qui se révèle être au fondement de nombreux écrits contemporains sur le jeu. Ce dernier montre comment, depuis le XIX ${ }^{e}$ siècle, le jeu présente dans certains cas une perméabilité avec le travail et le sérieux. Ces deux domaines sont pourtant traditionnellement tenus comme opposés aux activités ludiques, aujourd'hui encore ${ }^{3}$, lorsque le jeu s'inscrit par exemple comme une récréation dans l'espace éducatif. Pour Huizinga, cette collusion est notamment due au développement de la compétitivité économique liée à l'industrialisation et au développement des moyens de communication qui encourageraient le développement de certains «éléments purement ludiques » dans le travail :

Le développement de cet aspect agonal, qui entraine le monde du côté du jeu, a été favorisé par un facteur externe, au fond indépendant de l'esprit même de la culture; notamment l'extraordinaire perfectionnement des relations humaines dans tous les domaines, par tous les moyens. Technique, publicité et propagande encouragent partout la compétition, et rendent possible la satisfaction de cet instinct. [...] Jusqu'à une époque relativement récente, la compétition commerciale garde une allure primitive. Elle ne devient intense que grâce au trafic moderne, à la propagande commerciale et à la statistique. La notion de record, née dans le sport, n'allait pas manquer de gagner également du terrain dans la vie des affaires. [...] Partout où la production industrielle prenait un caractère sportif, l'aspiration au record se donnait carrière : le plus gros tonnage d'un paquebot, le ruban bleu pour le plus court trajet maritime. Ici un élément purement ludique a mis les raisons d'utilité tout à fait à l'arrière-plan: le sérieux devient jeu (Huizinga, 1938, pp. 319-320).

Dans la continuité de ces réflexions, et se référant explicitement à Huizinga, Joost Raessens a postulé que les technologies digitales semblent multiplier les éléments ludiques de la culture. Dans ce cadre, les téléphones mobiles, Internet et les jeux vidéo faciliteraient la construction d'identités ludiques chez les individus. En somme, ces technologies renforceraient la "ludification » de la culture, selon les termes employés par l'auteur. Bien que Raessens ne définisse pas précisément la notion de ludification, celle-ci a été par la suite utilisée à plusieurs reprises (Rao, 2008; White, Harviainen, Care Boss, 2012) pour qualifier une « tendance plus générale où le jeu (pas seulement le jeu vidéo, qui en fait partie bien sûr, mais le jeu en général, le ludique) prend une place de plus en plus importante dans la société aujourd'hui " (Picard, 2009). La difficulté soulevée par la notion de ludification est que ce terme est parfois employé en français comme une traduction de gamification, alors même que ces deux notions ne sont pas équivalentes dans la littérature anglophone - ce que le texte de Deterding \& al. met en évidence dans ce numéro (raison pour laquelle nous avons décidé de laisser en l'état les néologismes anglophones relatifs au jeu employés dans l'article).

Une autre difficulté soulevée par la notion de gamification, et qui a stimulé l'élaboration d'autres concepts, est l'adoption d'une approche essentialiste des phénomènes ludiques qu'elle postule; ce concept implique que certains éléments d'une situation de jeu seraient intrinsèquement ludiques et qu'il suffirait de les exporter à d'autres situations pour transformer ces dernières. On remarque alors que cette conception du jeu a déjà donné ailleurs matière à débat, en rappelant entre autres la posture adoptée par le philosophe du jeu Jacques Henriot. S'éloignant des définitions qu'il appelle 
«descriptives » du jeu (qui reposent sur une suite de critères fixes et immuables), Jacques Henriot avance qu'il y aurait bien un invariant anthropologique de jeu chez les humains (l'attitude ludique), mais que ceux-ci se feraient des idées distinctes de ce même phénomène (Henriot, 1989, pp. 83-84) : la conception du jeu et les objets qui renvoient à cette activité sont toujours susceptibles d'évoluer à travers le temps et l'espace. La multiplication et la mutation des phénomènes ludiques montrent que ces définitions sont elles aussi des constructions culturelles reflétant avant tout une certaine idée de ce qu'est cette « chose nommée jeu » (Henriot, 1989).

11 Cette approche du jeu comme phénomène mouvant, en perpétuelle transformation, générant sans cesse de nouvelles connotations, se retrouve de façon similaire dans les écrits de Thomas Malaby, notamment dans un article de 2007 intitulé «Beyond Play: A New Approach to Games " (qui néanmoins ne fait pas état des réflexions de Jacques Henriot, qui n'a jamais été traduit en anglais). Afin de prendre en considération ces phénomènes de transformation perpétuelle des caractéristiques des jeux, et pour sortir d'une perspective essentialiste, nous avons proposé la notion de ludicisation (Genvo, 2014), qui insiste entre autres sur les interactions qui s'établissent entre un contexte et une structure de jeu dans l'évolution des formes ludiques. Il s'agit dans ce cadre de décrire comment un objet qui n'était pas considéré comme un jeu en vient à être perçu comme tel et en quoi ce changement de perception peut conjointement mener à faire évoluer les acceptions du terme "jeu». Par exemple, Foursquare a un statut ambigu, alors que certains affirment qu'il s'agit d'une plateforme de socialisation et que d'autres vont revendiquer sa fonction ludique. Selon les concepteurs, «Foursquare vous aide à trouver les endroits parfaits pour sortir avec vos amis. Découvrir le meilleur de la bouffe, de la vie nocturne et du divertissement aux alentours ${ }^{4} »$. Or, l'utilisateur remarque aisément l'attrait ludique de l'application puisque les achats permettent désormais d'accumuler des points et de devenir «maire » du commerce en informant tous ses amis de cet accomplissement (les joueurs diraient "de cet achievement»). Foursquare répond en ce sens à la définition donnée par Zichermann et Cunningham de la gamification faisant de cette application de socialisation un jeu, comme si consommer et socialiser devaient désormais se faire sous la forme ludique.

\section{Les effets de la gamification}

12 Cet exemple, où la gamification est directement liées aux réseaux sociaux, n'est pas unique et nous démontre à quel point cette stratégie ludique peut avoir comme effet de modifier les comportements (notamment, en allant à certains endroits parce que nous accumulerons des points avec nos amis). La gamification peut alors imprégner les rapports sociaux de dimensions ludiques, en cela conforme à la tendance de persuasion «soft » identifiée par Lipovetsky (1983). Face au repli des grandes idéologies politiques, morales ou disciplinaires, cette tendance consiste à miser sur la gratification et le ludique au lieu d'autres types de rapports de pouvoir explicites. Du coup, s'introduisent de nouveaux rapports au pouvoir non seulement au sein des jeux, mais également dans les conduites et représentations des liens sociaux. Cette dynamique, qui n'est certes pas nouvelle, mais qui a pris de l'ampleur depuis l'avènement des technologies web, des plateformes mobiles et des applications, soulève de nombreuses questions éthiques qui se doivent d'être analysées en profondeur puisque le jeu semble s'immiscer dans toutes les sphères de la vie. En effet, faire de la course à pied peut désormais prendre la forme 
d'une fuite face à l'attaque de zombies grâce à l'application Zombies Run! ${ }^{5}$. Réaliser toutes les petites tâches du quotidien peut devenir l'occasion d'accumuler des points d'expérience et de faire progresser son avatar grâce à l'application EpicWin. ${ }^{6}$ Passer à travers une période difficile, par exemple pour accepter une maladie chronique ou se sortir de la dépression, peut désormais se faire à l'aide de SuperBetter ${ }^{7}$, un jeu développé par McGonigal (2012).

Toutes ces plateformes de gamification, qui participent de la ludicisation du quotidien, offrent une panoplie d'avantages que l'on peut résumer par la « motivation ». Les effets positifs sur le mode de vie des utilisateurs/joueurs, que ce soit en faisant de l'exercice de manière régulière, en réalisant de manière stimulante des tâches banales et répétitives ou en se sortant d'une dépression, sont louables et participent à un mieuxêtre collectif. Toutefois, il faut aussi garder en tête que cette gamification s'opère par le biais d'une approche béhavioriste de l'humain, où ces systèmes imposent une norme définie par les concepteurs du jeu. Cette normalisation peut à la fois être infantilisante et angoissante (par exemple, si les objectifs du jeu ne sont pas atteints « comme il se devrait»), mais elle peut aussi imposer un modèle idéologique sous le couvert du ludique. Si les processus de gamification profitent de l'aura du jeu en tant que mode de rapport positif, amusant et inoffensif, il n'en demeure pas moins que cette gamification se fait, pour l'instant, principalement sous l'hégémonie d'un système néolibéral qui profite de cet engouement.

14 En ce sens, cette gamification du monde fonctionne surtout grâce aux technologies numériques qui produisent une quantité astronomique de traces, traces d'identification des consommateurs qui se monnayent, entre autres sur le marché de la publicité, des systèmes de recommandation et des assurances. Le jeu devient alors un prétexte pour garder les individus connectés le plus longtemps possible, en produisant un maximum d'informations afin de peaufiner leur profilage. Les liens à faire entre la gamification et la surveillance sont nombreux (Whitson 2013; Whitson \& Simon, 2014) et, désormais, cette surveillance ludifiée traverse tous les pans de la vie sociale - comme en témoignent, par exemple, une majorité de jeux sur Facebook où l'on peut voir qui joue, à quels moments, à quel niveau du jeu, etc. Il faut ajouter que cette production de traces se fait en partie de manière volontaire, tandis qu'une panoplie d'applications ludiques encouragent la diffusion d'informations à laquelle participent les usagers de manière motivée et transparente. Cette acceptation du partage de ses propres informations se fait en échange d'une expérience ludique qui a comme objectif de rendre la vie plus amusante. Toutefois, ce mode de rapport au monde se fait au prix, bien caché et souvent opaque, d'un contrôle social où le comportement des individus est "dressé " par l'usage de stratégies ludiques, alors que le « joueur » doit s'adapter à une structure la plupart du temps très rigide comparée à des règles d'un jeu qui devrait, par définition, être toujours socialement négociables.

De la même manière, sous le couvert d'un milieu de travail rendu ludique, certaines compagnies usent de stratégies de gamification pour « motiver » leurs employés (parfois simplement avec l'accumulation de points ou de badges), mais avec l'objectif principal d'augmenter leur productivité en mettant les travailleurs en compétition envers euxmêmes ou les uns envers les autres. L'application RepTivity ${ }^{8}$ est un exemple frappant de ludification agonistique des performances des vendeurs dont le fonctionnement est de publiciser le nom des " gagnants » : «Avec RepTivity, les entreprises peuvent classer les représentants des ventes en se basant sur des indicateurs clés de la performance et en 
rendant public les meilleurs vendeurs d'une façon qui demeure agréable pour tous ${ }^{9}$ ». Cette façon de stimuler la productivité est reprise par DueProps ${ }^{10}$ qui fonctionne sur la base de la reconnaissance par les collègues de la valeur du travailleur parce que "lorsque les gens se sentent appréciés, ils sont plus heureux, s'engagent davantage et deviennent plus productifs ${ }^{11}$ ». Encore une fois, avec ces exemples, on favorise, sous le couvert du jeu, une vision du système économique basé sur l'accumulation, l'efficience et la productivité. Plus simplement, il faut se demander si la gamification, utilisée dans le contexte marchand, n'est pas en train de transformer l'idée de jeu (Bonenfant, 2013).

Comme on peut le constater, penser les processus de transformation et de contagion du jeu engage à mener tant des réflexions ontologiques, méthodologiques, épistémologiques qu'éthiques, le choix des concepts mobilisés n'étant pas tout à fait neutre dans les réponses que l'on va apporter à ces différents points. Ceci montre néanmoins que la relation entre les différentes notions de gamification, ludification et ludicisation, leur perméabilité ou dissemblance, reste une question vive qui demande à être analysée pour comprendre et mettre en évidence « ce qui fait jeu » et quels en sont les enjeux. C'est ce que propose de faire chacun des textes composant le dossier de ce numéro, qui investissent chacun à leur façon cette problématique à partir de terrains hétérogènes. Fanny Barnabé aborde l'intégration du ludique au sein des pratiques de création littéraire (par l'intermédiaire des fans-fictions); Françoise Lejeune analyse quant à elle le possible rôle de la gamification lors d'une expérimentation artistique (à partir du cas des jeux en réalité augmentée); finalement, Victor Potier présente la façon dont le jeu est mobilisé comme mode de gestion des relations en entreprise. Du fait de ces différents terrains, ces réflexions permettent de restituer des logiques que recouvrent dans nos sociétés les phénomènes de mise en forme ludique, qu'il s'agisse de les comprendre comme entreprise de rationalisation d'activités par l'ajout de contraintes du jeu (inhérentes au "game») ou d'invitation à une liberté créatrice (que l'on retrouve au centre du " play»).

\section{BIBLIOGRAPHIE}

BOGOST I. (2007), « Persuasive Game: Explotationware », gamasutra.com, en ligne : http:// www.gamasutra.com/view/feature/6366/persuasive_games_exploitationware.php?page=4 BONENFANT M. (2013), « Gamification et marketing : rôle des stratégies de ludification en ligne dans les processus de reconnaissance identitaire », World Social Science Forum/ World Social Science Forum, Montréal, 13-15 octobre.

COOPER \& al. (2010), « Predicting Protein Structures With a Multiplayer Online Game », Nature, $\mathrm{n}^{\circ} 466$, pp. 756-760.

COLL S. (2014), Surveiller et récompenser: Les cartes de fidélité qui nous gouvernent (préface de David Lyon), Zürich, Ed. Seismo, Collection Terrains des sciences sociales. 
DETERDING S. (2010), « Pawned. Gamification and its Discontents », Playful 2010, London, 24 Septembre, en ligne : http://blog.jointhecompany.com/2010/09/29/pawned-gamification-andits-discontents/

DETERDING S. (2011), « A Quick Buck by Copy and Paste », gamification-research.org, 2011, en ligne : http://gamification-research.org/2011/09/a-quick-buck-by-copy-and-paste

DETERDING S., DIXON D., KHALED R. \& NACKE L. (2011), « From Game Design Element to Gamefulness: Defining Gamification", Proceedings of the 15th International Academic MindTrek Conference, ACM, pp. 9-15.

FUCHS M., FIZEK S., RUFFINO P., SCRAPE N. (2014) (dirs.). Rethinking Gamification, Meson Press, Lüneburg.

GENVO S. (2014), Penser la formation et les évolutions du jeu sur support numérique, Mémoire d'habilitation à diriger les recherches, Université de Lorraine, en ligne : http:// www.ludologique.com/wordpress/?p=445

HENRIOT J. (1989), Sous couleur de jouer, Paris, José Corti.

HUIZINGA J. (1938), Homo ludens. Essai sur la fonction sociale du jeu, Trad. Seresia C., Paris, Gallimard, réed. Gallimard, 1951.

LIPOVETSKY G. (1983), L’ère du vide: essais sur l'individualisme contemporain, Paris, Gallimard.

MALABY T. M. (2007), « Beyond Play. A New Approach to Games », Games and culture, n², pp. 95-113.

MCGONIGAL J. (2011), Reality is Broken: Why Games Make Us Better and How They Can Change the World, Penguin Books.

PICARD M. (2009), « Les influences mutuelles du cinéma et du jeu vidéo », Entretien pour parolecitoyenne.org, en ligne : http://parolecitoyenne.org/blogs/wp-content/uploads/2009/02/ cinema-et-jeu-video-m-picard.pdf

RAESSENS J. (2006), « Playful identities, or the ludification of culture », Games and Culture, Vol. $1(1)$, pp. 52-57.

RAO V. (2008), « Facebook Applications and Playful Mood: the Construction of Facebook as a "Third Place" ", Proceedings of the 12th international conference on Entertainment and media in the ubiquitous era, pp. 8-12.

WHITE W. J., HARVIAINEN T., CARE BOSS E. (2012), « Role-Playing Communities, Cultures of Play and the Discourse of Immersion », in: Torner E., White W. J. (dirs), Immersive Gameplay: Essays on Participatory Media and Role-Playing, McFarland \& Company, Inc.

WHITSON J. (2013), « Gaming the Quantified Self », Surveillance \& Society, vol. 11, n 1/2, pp. 163-176.

WHITSON J. \& SIMON B. (dir.) (2014), « Surveillance, Gaming and Play », Surveillance \& Society, vol. $12, \mathrm{n}^{\circ} 3$.

ZICHERMANN G. \& CUNNINGHAM C. (2011), Gamification by design, Sebastopol, O’Reilly Media.

\section{NOTES}

1. http://progresswars.com/

2. Notre traduction. 
3. Jacques Henriot remarque à ce sujet qu' ' aucune antinomie ne paraît plus classique, plus fondamentale que celle-là. Au plaisir que prend le joueur, on oppose la peine qu'éprouve le travailleur; à la liberté du jeu, la contrainte du travail. Les enfants eux-mêmes, que leur entourage initie très tôt à la pratique de cette distinction, répondent à toute question concernant le contraire du jeu: le travail. [...] Elle appartient aussi à un ensemble idéologique, à une conception de l'homme et du monde» (Henriot, 1989, p.194).

4. Traduction libre : «Foursquare helps you find the perfect places to go with friends. Discover the best food, nightlife, and entertainment in your area», www.foursquare.com (consulté le 03/09/14).

5. http://www.zombiesrungame.com (consulté le 03/09/14).

6. https://itunes.apple.com/ca/app/epicwin/id372927221?mt=8 (consulté le 03/09/14).

7. https://www.superbetter.com/ (consulté le 03/09/14).

8. http://www.reptivity.com/ (consulté le 03/09/14).

9. Traduction libre: "With RepTivity, organizations can rank their sales reps based on key performance indicators and publicly recognize sales leaders in a format everyone can enjoy. ".

10. https://dueprops.com/ (consulté le 03/09/14).

11. Traduction libre : "when people feel appreciated, they're happier, more engaged and more productive $»$.

\section{AUTEURS}

\section{MAUDE BONENFANT}

Université du Québec à Montréal

\section{SÉBASTIEN GENVO}

Université de Lorraine 